\title{
el coeficiente adimensional de robustez en el călculo de vigas
}

Sincoping
Se propone y discute el concepto de ROBUSTEZ, como medida de la resistencia a esfuerzos imprevistos. Se hace una aplicación al distanciamiento más económico entre las vigas principales de un entramado horizontal para cubiertas o forjados.

Se ve la gran utilidad del concepto propuesto, al obtener directamente el precio de un entramado resistente, sin necesidad de proyectarlo.

Se aplica, por último, la robustez al costo de puentes de hormigón pretensado.

\section{Sirmbolos untillizodos}

$\left.\begin{array}{l}A \\ B\end{array}\right\}=$ escalas de nomograma

$b \quad=$ ancho de viga rectangular

$C=$ constante

c $=$ canto (subíndice para correas)

$D$ = diámetro

$e \quad=$ espesor

$E \quad=$ módulo elástico

$F$ = área transversal de una sección

$h$ = subíndice de cercha

$I \quad=$ momento de inercia

$K \quad=$ constante de material $\left(\gamma / \sigma^{2 / 3}\right)\left(K^{\prime}\right.$ es para material distinto del acero $)$

$L\}=$ luz

$m$ = coeficiente de aumento de la sobrecarga, debida al peso unitario de la viga

$M \quad=$ momento flector

$N \quad=$ número de correas

$p \quad=$ precio por $\mathrm{m}^{2}$ de la estructura

$p \quad=$ peso por $\mathrm{m}$. l. de un perfil o viga

$q=$ sobrecarga total ( $q_{t}=$ peso del tablero; $q_{i}=$ sobrecarga equivalente al tren móvil)

$R \quad=$ robustez

$r \quad=$ radio de giro $(\sqrt{I / F})$

$S \quad=$ separación entre cerchas o vigas principales

$V \quad=$ separación entre correas

$W=$ módulo resistente $\frac{2 \cdot I}{c}$

$Z$ = escala de nomograma, producto de 4 variables (fig. 3) ( $Z^{\prime}$, corrección por material distinto al del ábaco) 
$\alpha \quad=$ coeficiente adimensional para correas continuas $(1,17)$

$\beta \quad=$ coeficiente adimensional que mide la esbeltez $(c / \sqrt{ } \cdot \bar{F})$ de una sección

$\gamma \therefore=$ peso específico

$\delta \quad=$ precio por $\mathrm{kg}$ del acero, incluido el montaje

$\eta$. = rendimiento de material a flexión pura

$\lambda \quad=$ relación de precios de dos materiales (incluido montaje)

$\mu=$ coeficiente adimensional $\left(\lambda \cdot \frac{K_{h}}{K_{c}}\right)$ para el caso de emplear materiales distintos

$\varrho \quad=$ coeficiente adimensional $\left(\frac{r}{c}\right)$ que compara el radio de giro con el canto

$\sigma:=$ carga de trabajo del material

$\vartheta \quad=$ coeficiente de reparto transversal

\section{Rendîmiento segưn Ia forma de unce secoión simmetrick y homogenen}

En una viga trabajando a flexión, dentro de la hipótesis de Navier sobre deformación elástica y plana de la sección normal, el momento admisible viene dado por:

$$
M=2 \frac{\sigma}{c} \cdot I
$$

(llamando $\sigma$ a la carga de trabajo; $c$ al canto; $I$ al momento de inercia), es decir:

$$
\frac{M}{\sigma}=2 F \cdot \frac{r^{2}}{c}
$$

(siendo $F$ sección, y $r$ el radio de giro).

Es muy interesante comparar, para cada forma geométrica de la sección, el costo (que es proporcional al área $F$ ) con el momento máximo que puede soportar, o mejor aún, independientemente de la carga de trabajo, con el módulo resistente $W=\frac{M}{\sigma}$.

Las dimensiones de estas variables son $L^{2}$ y $L^{3}$. Su cociente, $W / F$, significa la capacidad de resistir momentos por unidad de área, y puede llamarse rendimiento geométrico de la sección. La dimensión resultante es una longitud.

Este rendimiento,

$$
\eta=\frac{M}{\sigma F}=2 r \cdot\left(\frac{r}{c}\right)
$$

depende del radio de giro.

En una sección, la mejor distribución de áreas es la que resulta de alejarlas lo más posible de la fibra neutra. Así se consigue el mejor rendimiento del material. Puede considerarse a la inversa, que un mismo momento puede soportarse con áreas tan pequeñas como se quiera, suficientemente alejadas de la fibra neutra.

Dos secciones geométricamente semejantes no tendrán igual rendimiento, pues hemos visto que depende del tamaño. 
Si interesaran los esfuerzos transversales -momentos no centrados- se estudiará el radio de giro transversal, con igual conclusión. Euler, en las fórmulas de pandeo, relaciona el radio de giro y la luz libre. Resulta, para cargas axiles, un número sin dimensiones:

$$
\frac{P}{F E}=C \cdot \frac{r^{2}}{l^{2}}=\frac{\sigma}{E} .
$$

Se ve que $\sigma / E$ es como un coeficiente de seguridad, función de la forma geométrica de la sección y de la luz entre apoyos. Para estudiar el pandeo es lógico comparar la forma de la sección con la luz libre.

Obsérvese que pueden conseguirse radios de giro grandes, en todas las orientaciones, disponiendo las áreas activas alejadas de la fibra neutra en todas las direcciones, es decir, un conjunto de cordones muy separados unos de otros.

Se obtiene así, como sección ideal, una configuración de cordones dispersos y de sección muy débil. Todo lo dicho hasta ahora para flexión o pandeo es igualmente válido para ejes a torsión, llegándose a la misma conclusión: el rendimiento óptimo del material consiste en disponer pequeñas áreas activas, dentro de cada sección, muy alejadas de la fibra neutra. Por este camino se pasa de la estructura clásica de celosía a la de redondos soldados, que forman unidad mediante arriostramientos también débiles.

Ocurre, sin embargo, que con frecuencia se olvidan algunas realidades del trabajo de estas piezas, como son los golpes, las cargas aisladas y otras solicitaciones a que la estructura se ve sometida en el proceso de montaje, tanto de sí misma como de elementos con ella relacionados (grúas, maquinaria, etc.)

Debe por ello matizarse convenientemente la determinación del rendimiento, ya que, de no hacerse así, podrían resultar soluciones peligrosas y débiles, pese a la corrección del proyecto para soportar los esfuerzos convencionales de flexión pura y centrada.

Un fallo de soldadura, un pandeo parcial o un golpe, repercuten en toda la estructura; resultan, por tanto, soluciones delicadas que pueden llamarse de «riesgo total».

\section{Robustex: función de forma}

En la comparación de proyectos, no deben equipararse soluciones de distinto riesgo, cuyos precios, naturalmente, serán distintos, debido a que también será diferente su coeficiente real de seguridad.

El límite que proponemos para esta búsqueda del rendimiento debe ser un coeficiente de forma, sin dimensiones por tanto, que indique la concentración de áreas activas en torno a la fibra neutra, y que relacione $2 \cdot \frac{I}{c}$ y $F$.

En rigor, como Euler hace para el pandeo, cabe pensar que debe también figurar la luz libre en esta relación. Sin embargo, intuitivamente el proyectista prescinde a menudo de la luz. Si lo que preocupa son los golpes y cargas aisladas, utiliza secciones relativamente robustas.

Por otra parte, es muy útil tener relacionados el peso de un perfil y el momento que puede soportar, como veremos luego, mediante un coeficiente de forma que se sepa determinar. 
La única combinación adimensional entre $2 \cdot \frac{I}{c}$ y $F$ es del tipo:

$$
R=\frac{F}{(2 I / C)^{2 / 3}}=\frac{F}{(M / \sigma)^{2 / 3}} .
$$

A esta expresión la llamaremos robustez de la sección. Su sentido aparece claro al estudiar la fórmula del rendimiento del material,

$$
\eta=\frac{M}{\sigma F}=2\left(\frac{r}{c}\right)^{2} \cdot c,
$$

y al expresar el canto en función de alguna longitud representativa del tamaño de la sección. Elegiremos el lado del cuadrado de área equivalente $\sqrt{F}$, ya que interesa, sobre todo, el peso por metro de viga. Entonces:

$$
\frac{\eta}{\sqrt{\bar{F}}}=\frac{M / \sigma}{F^{3 / 2}}=2\left(\frac{r}{c}\right)^{2} \cdot \frac{c}{\sqrt{\bar{F}}}=2 \varrho^{2} \cdot \beta,
$$

con lo que:

$$
R=\frac{F}{(M / \sigma)^{2 / 3}}=\left(\frac{1}{2 \beta \cdot \varrho^{2}}\right)^{2 / 3} .
$$

La robustez de una sección mide, en realidad, su esbeltez $\left(\beta=\frac{c}{\sqrt{F}}\right)$ y la posición relativa del centro de inercia de las áreas activas $\left(\varrho=\frac{r}{c}\right)$.

Puede trabajarse con este concepto de robustez, igual que con un coeficiente de seguridad adoptado antes del proyecto, que se elegirá considerando las distintas circunstancias de cada estructura. Por debajo de un cierto valor crítico de la robustez, incluso con independencia de la luz, las vigas o cerchas no ofrecen seguridad contra golpes locales y pandeos. Aunque estén bien calculadas a flexión pura, estas estructuras son de «riesgo total». En ellas, el fallo de algún elemento resistente por solicitaciones ajenas a las hipótesis convencionales de carga es bastante verosímil dada su debilidad relativa, y, como consecuencia de tal fallo, puede provocarse un colapso del conjunto sin haberse rebasado las cargas de trabajo clásicamente consideradas.

Queremos insistir en que, distintos proyectos, sólo resultan realmente comparables si tienen robusteces parecidas. Precisamente la eleccion de esta magnitud constituye una de las manifestaciones más concretas del criterio del ingeniero director del proyecto.

\section{Recomenducioness pura secciomes metúlicas}

En la figura 1 se han representado las secciones, pesos y momentos admisibles, según el material, de las formas resistentes clásicas.

Se observa que los perfiles normales (I) tienen robusteces cercanas a la unidad (cada robustez es una línea de pendiente 2/3). Los perfiles de ala ancha tienen robusteces de 1,25 y pueden llegar a 2,2 usando platabandas.

Las vigas de alma llena compuestas con angulares se mueven entre valores de la robustez de 0,5 y 0,6 ; y las de chapa soldada, entre 0,3 y 0,5 . 


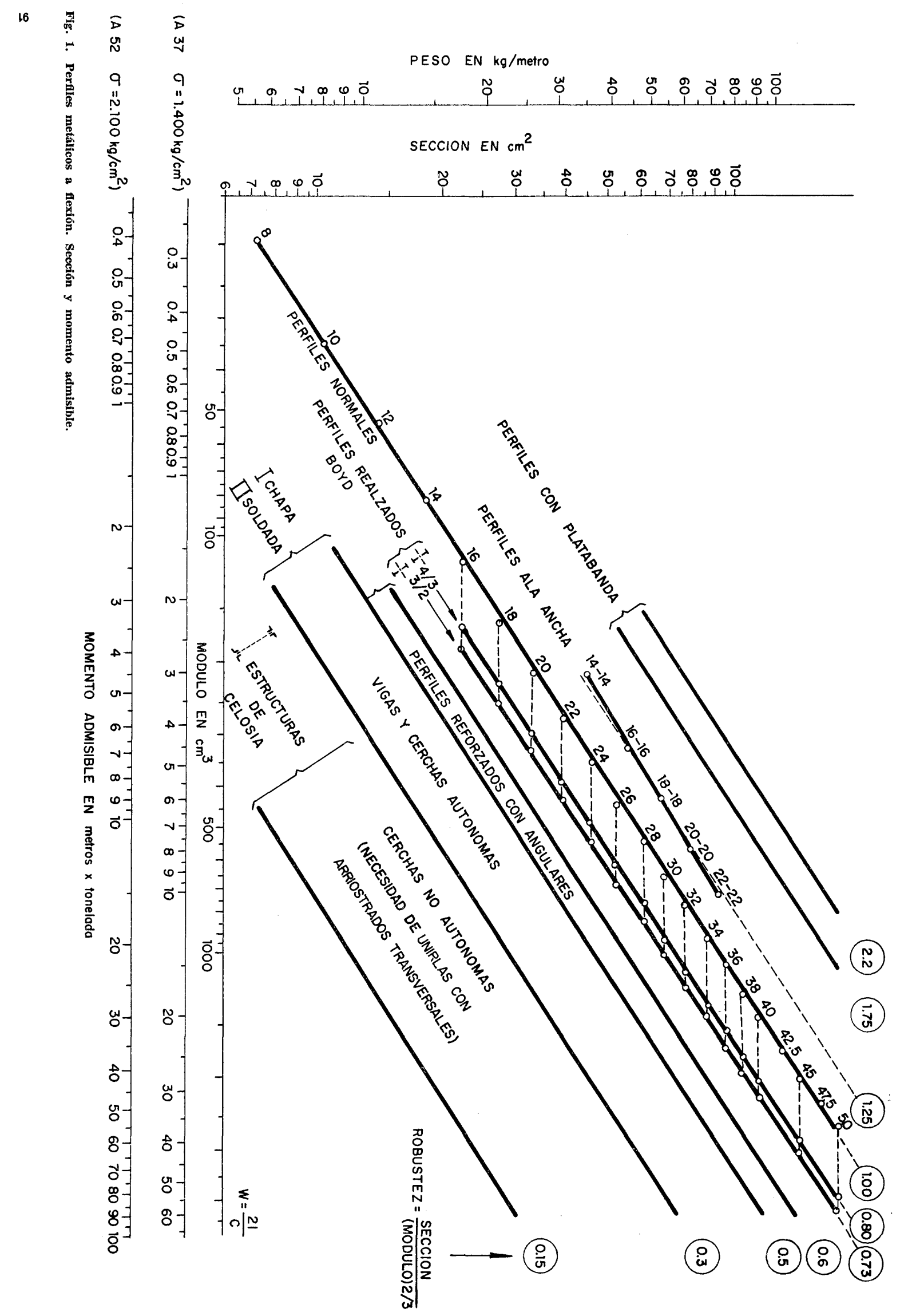


Se propone en los cuadros números 1 y 2 las robusteces convenientes según el destino de las estructuras para flexión pura o compuesta, respectivamente:

\section{CUADRO NUM. 1. Robusteces recomendadas para flexión pura}

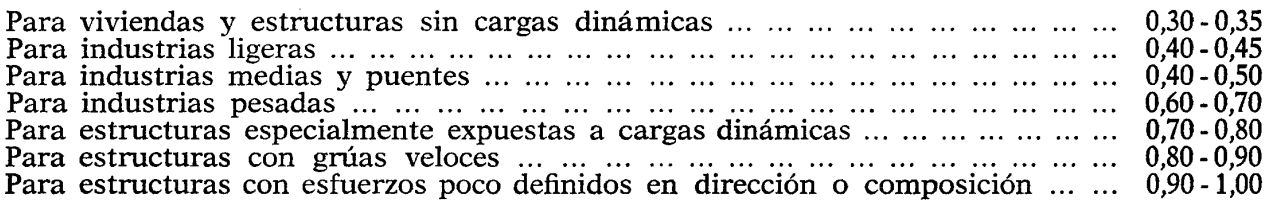

\section{CUADRO NUM. 2. Robusteces recomendadas para flexión compuesta}

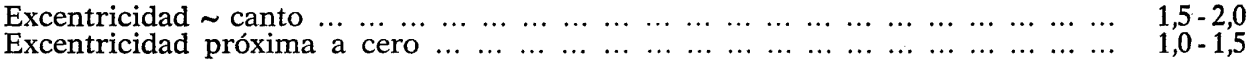

\section{Apulicuciones}

Una de las ventajas principales del concepto de robustez, y del hecho de poderse fijar previamente al diseño de la estructura, es que proporciona una relación entre el momento y la sección, es decir, el peso de la viga.

Aplicaremos este método a casos muy distintos, donde resulta manifiesta su utilidad.

Entramados horizontales (forjados y cubiertas): Llamaremos $p$ al peso por metro lineal de una viga o cercha ( $\gamma$ es el peso específico del material). Las secciones son homogéneas y simétricas.

Por definición de robustez:

donde

$$
p=\gamma \cdot S=\gamma \cdot R \cdot\left(\frac{M}{\sigma}\right)^{2 / 3}=\left(\frac{\gamma}{\sigma^{2 / 3}}\right) \cdot M^{2 / 3} \cdot R=K \cdot R \cdot M^{2 / 3},
$$

$$
K=\left(\frac{\gamma}{\sigma^{2 / 3}}\right)
$$

depende del material y de las unidades empleadas. En lo que sigue el momento vendrá en $\mathrm{m} \cdot \mathrm{kg}$. Para el acero $\gamma=7.800 \mathrm{~kg} / \mathrm{m}^{3}\left(^{*}\right)$, entonces, respectivamente, para acero A37 ( $\sigma=$ $\left.=1.400 \mathrm{~kg} / \mathrm{cm}^{2}\right)$ y $\mathrm{A} 52\left(\sigma=2.100 \mathrm{~kg} / \mathrm{cm}^{2}\right)$ :

$$
K_{37}=0,134 \quad \text { y } \quad K_{52}=0,102 .
$$

Llamaremos $V$ a la separación entre correas, $S$ la de las cerchas, $L$ es la luz de la nave, que viene definida por el destino de ésta. También $V$ lo está por el material de cierre que vaya a emplearse. Normalmente debe elegirse una separación $S$. Para ello utilizaremos el concepto de peso de estructura por unidad de área cubierta. Llamaremos $q$ a la sobrecarga por $\mathrm{m}^{2}$ (peso del cierre + viento + etc.). Los subíndices $c$ y $h$ corresponden a correas y cerchas, respectivamente.

(*) Para el hormigón pretensado: $K=0,22$, con $\sigma=120 \mathrm{~kg} / \mathrm{cm}^{2}$.

Para el aluminio: $K=0,12$, con $\sigma=300 \mathrm{~kg} / \mathrm{cm}^{2}$. 
En las correas el momento vale:

$$
M_{c}=\left(p_{c}+q \cdot V\right) \cdot \frac{S^{2}}{8}, \quad \text { o bien } \quad M_{c}=\frac{m_{c}}{8} \cdot q \cdot V \cdot S^{2}
$$

(o partido por 10 , si son continuas).

Llamando $m$ a la influencia relativa del peso propio por $\mathrm{m}^{2}$ de nave, tenemos:

$$
m_{c}=\left(1+\frac{p_{c}}{q \cdot V}\right)
$$

(que suele valer $1,0-1,1$ ).

Utilizando el coeficiente $\alpha$ que vale normalmente 1 para correas apoyadas, o bien $\alpha=1,17$ para correas continuas, el peso por $\mathrm{m}^{2}$ de nave de las correas es [1]:

$$
\frac{p_{c} \cdot S}{S \cdot V}=\frac{p_{c}}{V}=\frac{m_{c}^{2 / 3}}{4 \alpha} \cdot K_{c} \cdot R_{c} \frac{q^{2 / 3} \cdot S^{4 / 3}}{V^{1 / 3}} .
$$

En las cerchas, análogamente:

$$
\frac{p_{h} \cdot L}{L \cdot S}=\frac{m_{h}^{2 / 3}}{4} \cdot K_{h} \cdot R_{h} \frac{q^{2 / 3} \cdot L^{4 / 3}}{S^{1 / 3}}
$$

(sólo consideraremos las cerchas apoyadas),

con

$$
m_{h}=\left(1+\frac{p_{h}}{q \cdot S}\right)
$$

(que suele oscilar entre $1,1-1,3$ ).

En general, $R_{h}, R_{c}$ y lo mismo $K_{h}$ y $K_{c}$ serán distintas en correas y cerchas, pues pueden emplearse distintos materiales.

El precio total de entramado por metro cuadrado de cubierta $P$, será la suma de estos dos pesos unitarios, $\frac{p_{c}}{V}$ y $\frac{p_{h}}{S}$, multiplicados por los precios del acero de las correas, $\delta$ y del de las cerchas $\delta^{\prime}$, incluido su montaje (que oscila entre el 50 y el 100 por 100 del material) $\left({ }^{*}\right)$.

Sacando factor común $\delta$, y llamando $\lambda$ a la relación de precios $\frac{\delta^{\prime}}{\delta}$ queda:

$$
\frac{P}{\delta \cdot L}=\frac{R_{c} \cdot K_{c}}{4} \cdot q^{2 / 3} \cdot m_{c}^{2 / 3} \cdot\left[\left(\frac{L}{V}\right)^{1 / 3} \cdot\left(\frac{S}{L}\right)^{4 / 3} \cdot \frac{1}{\alpha}+\lambda \cdot \frac{K_{h}}{K_{c}} \cdot \frac{R_{h}}{R_{c}} \cdot\left(\frac{L}{S}\right)^{1 / 3} \cdot\left(\frac{m_{h}}{m_{c}}\right)^{2 / 3}\right]
$$

Separación óptima entre cerchas: Derivando respecto de $\left.\frac{S}{L}{ }^{* *}\right)$ se observa que esta función presenta un mínimo para el costo total, que resulta independiente de la carga $q$; su expresión será:

$$
\frac{S}{L}=\left(\frac{\alpha \cdot \lambda}{4} \cdot \frac{K_{h}}{K_{c}} \cdot \frac{R_{h}}{R_{c}}\right)^{3 / 5} \cdot\left(\frac{V}{L}\right)^{1 / 5} \cdot\left(\frac{m_{h}}{m_{c}}\right)^{2 / 5}
$$

(*) Si se trata de hormigón pretensado, $\delta$ ó $\delta^{\prime}$ son los precios del kilo de este material, ya montado.

(**) Despreciamos la variación de $\left(\frac{m_{h}}{m_{c}}\right)^{2 / 3}$, que es pequeña, como veremos. Incluyendo la derivada de esta variable, para la obtención del mínimo, puede verse que los errores son menores del 5 por 100. 


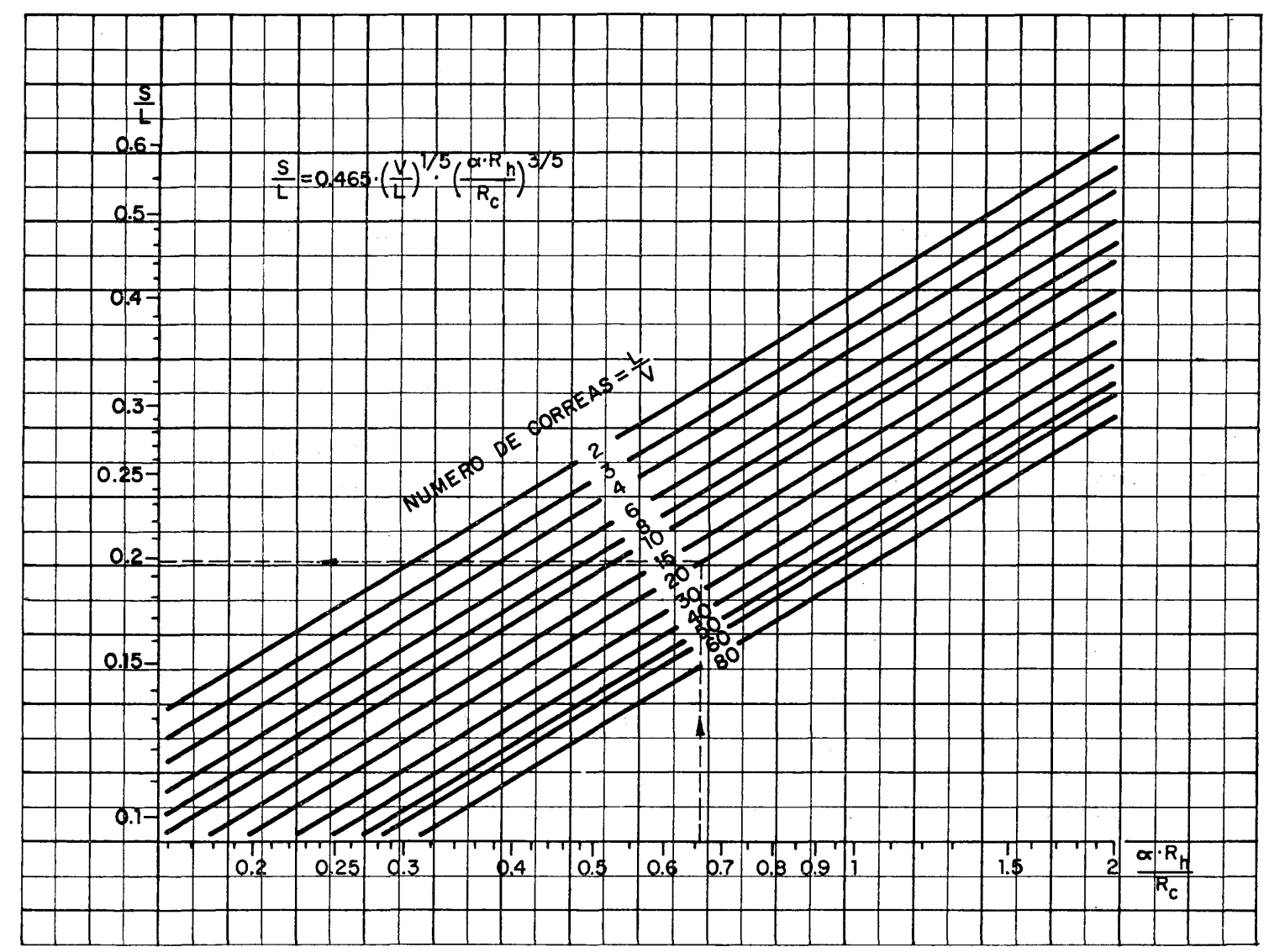

Fig. 2. Abaco para la separación óptima entre cerchas.

Obsérvese que el significado de este mínimo es que el costo de las correas debe ser el 20 por 100 del costo total. (Es decir, que la relación de los dos sumandos sea de $1: 4$ ).

El factor $\left(\frac{m_{h}}{m_{c}}\right)^{2 / 5}$ no es conocido, pero veremos más tarde que su influencia no llega a ser del orden del 10 por 100. Sin embargo, si utilizamos su valor medio y más probable $(1,065)$ podemos asignarle la condición de factor constante con errores menores del 5 por 100 . Esto es más que suficiente para $S$, ya que debe ser un submúltiplo de la longitud de la nave. Como en la mayor parte de las aplicaciones la estructura estará construida con el mismo material en correas y cerchas, también puede simplificarse, de momento, el factor $\mu=\lambda \cdot K_{h} / K_{c}$. Estudiaremos después el caso en que se empleen distintos materiales.

Si $\mu=1$, la separación es la siguiente:

$$
\frac{S}{L}=0,465 \cdot\left(\frac{V}{L}\right)^{1 / 5} \cdot\left(\frac{\alpha \cdot R_{h}}{R_{c}}\right)^{3 / 5}
$$

Con el ábaco de la figura 2 se obtiene gráficamente este valor.

Este mínimo económico lleva a separaciones de cerchas muy distintas de las usuales en naves industriales, debido a que suele suponerse que cada cercha necesita apoyar en columna. 
El costo de éstas y el estorbo que ocasionan entre dos naves contiguas, aconsejaban dar a las cerchas una separación mayor que el valor de [4]. Sin embargo, el presente estudio ha nacido de la utilización de la viga corrida (necesaria para el puente-grúa), como apoyo de las cerchas, que se independizan así de las columnas.

Determinación del costo mínimo, previa al proyecto: Sustituyamos ahora el valor óptimo $\frac{S}{L}$ obtenido en [3] con completa generalidad, en la fórmula [2] del precio de la estructura por metro cuadrado de nave; se tendrá como precio mínimo:

$$
P=4,15 \times 10^{-3} \cdot L \cdot q^{2 / 3} \cdot\left(\frac{L}{V}\right)^{1 / 15} \cdot\left(\frac{R_{c} \cdot K_{c}}{\alpha}\right)^{1 / 5} \cdot\left(R_{h} \cdot K_{h} \cdot \lambda\right)^{4 / 5} \cdot m_{h}^{8 / 15} \cdot m_{c}^{2 / 15},
$$

es decir:

$$
P=L \cdot \delta \cdot \sqrt{m_{h}} \cdot \mu^{4 / 5} \cdot Z
$$

donde:

$$
Z=0,054 \cdot q^{2 / 3} \cdot\left(\frac{L}{V}\right)^{1 / 15} \cdot R_{c}^{1 / 5} \cdot R_{h}^{4 / 5}
$$

Este valor de $Z$ puede obtenerse del nomograma de la figura 3 del modo siguiente: se partirá de la sobrecarga $q$ y del número de correas $\frac{L}{V}$ ( $V$ depende -como dijimos- del tipo de material utilizado en la cubierta); se obtiene así un punto en la escala $A$. Por otra parte, de las robusteces fijadas de antemano se obtiene otro punto en la escala $B$. La unión de ambas determina el valor de $Z$.

Al igual que antes, $\mu$ será, casi siempre, la unidad $\mathrm{y}$, por ello, no hemos querido complicar el nomograma incluyendo esta variable. Veremos que basta corregir con un coeficiente los resultados obtenidos del gráfico, en el caso de emplear materiales distintos.

También hemos simplificado la influencia de $m_{c}$ y $\alpha$, que es muy pequeña, sustituyéndolos por sus valores medios (coinciden en 1,07). Con ello se obtienen, como máximo, errores del orden del 2 por 100. Por último, hemos dado a $K_{c}$ el valor 0,134, que es el que corresponde al acero A37 corrientemente utilizado en la construcción de las correas. Cabe, sin embargo, emplear el nomograma para cualquier otro material y, en este caso, debe corregirse la $Z$ obtenida gráficamente, pues $Z^{\prime}$ es directamente proporcional a este valor de $K_{c}$ :

$$
Z^{\prime}=\frac{K_{c}^{\prime}}{0,134} \cdot Z_{\mathrm{A} 37}
$$

La influencia del peso propio de las cerchas es $m_{h}^{8 / 15} \approx \sqrt{m_{h}}$. La condición de separación óptima $S$ obligaba a que el costo de las cerchas fuera el 80 por 100 del total.

Entonces:

$$
\frac{0,8}{\lambda} \cdot \frac{P}{\delta \cdot q}=\frac{0,8 \cdot L \cdot Z \cdot \sqrt{m_{h}}}{\lambda \cdot q}=\frac{P_{h}}{q \cdot S}=m_{h}-1 .
$$

Resolviendo la ecuación y despreciando los términos pequeños, se obtiene:

$$
\begin{aligned}
& m_{h} \simeq 1+\frac{0,8 \cdot L \cdot Z}{q} \\
& m_{c} \simeq 1+\frac{0,2 \cdot L \cdot Z}{q} .
\end{aligned}
$$




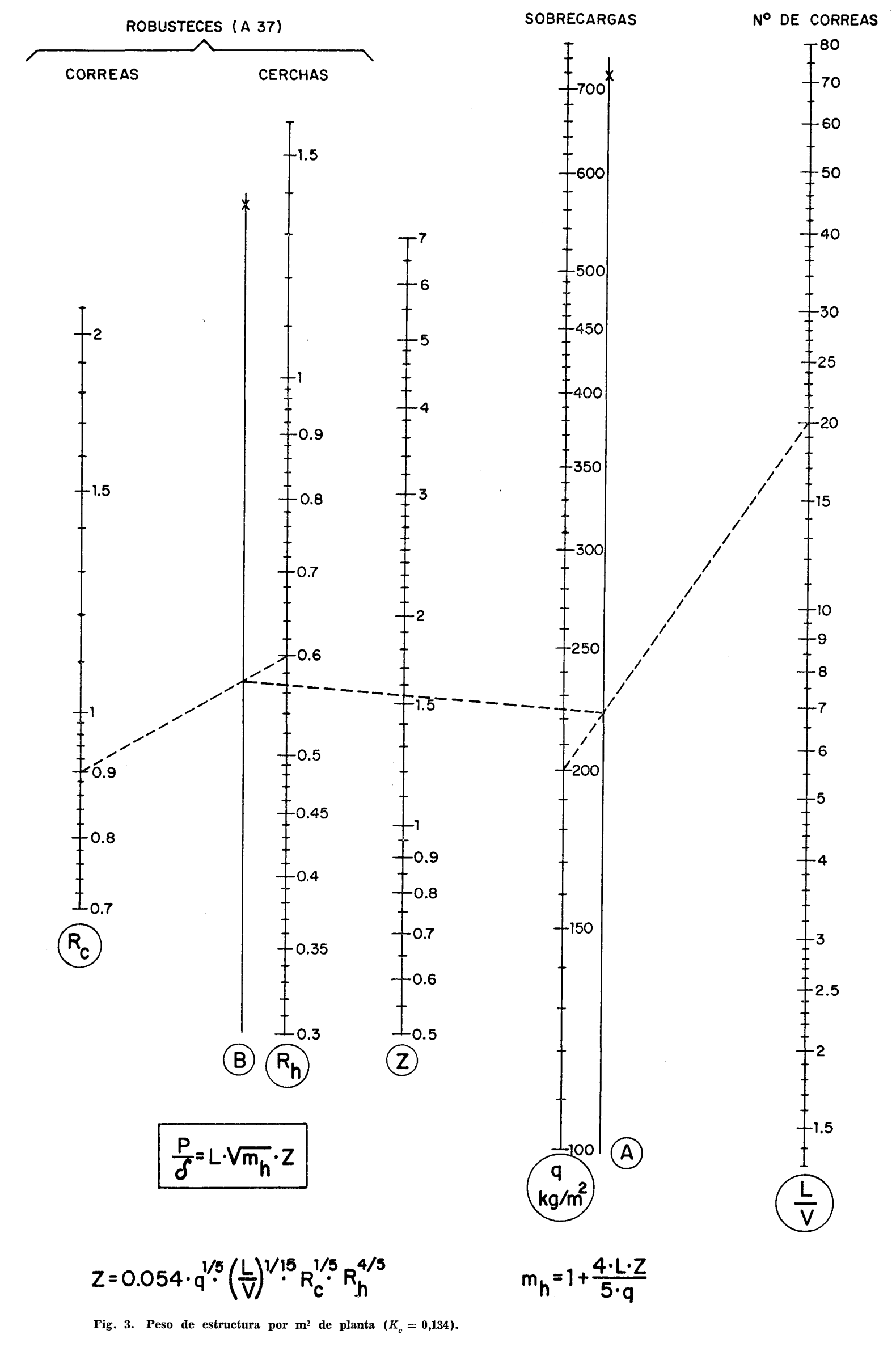


Podemos ver ahora que:

$$
\frac{m_{h}}{m_{c}} \simeq 1+\frac{0,6 \cdot L \cdot Z}{q},
$$

que para acero, luces menores de $40 \mathrm{~m}$ y $q$ mayores de $100 \mathrm{~kg} / \mathrm{m}^{2}$, nunca llega a valer 1,20 .

El proceso del cálculo es como sigue: se obtiene la $Z$ gráficamente (fig. 3) y de este valor y la fórmula [7] tendremos $m_{h}$. Se aplica entonces la fórmula [5], que normalmente $(\mu=1)$ se reduce a

$$
\frac{P}{\delta}=\sqrt{m_{h}} \cdot L \cdot Z,
$$

y representa el peso de acero por $\mathrm{m}^{2}$ de nave, proyectado con la condición de $S_{\text {opt }}$ dada por [2].

Ejemplo: Aplicaremos estos gráficos al anteproyecto de una nave de $30 \mathrm{~m}$ de luz, con cierre de hormigón pretensado $(V=1,5 \mathrm{~m})$ y correas (A37), apoyadas $(\alpha=1)$ de robustez 0,9 (perfiles I). Las cerchas (A37) de alma llena deberán tener $R_{h}=0,6$. La sobrecarga es $q=200 \mathrm{~kg} / \mathrm{m}^{2}$.

Entonces $\mu=1 ; \frac{L}{V}=20$ y resulta $Z=1,54$, como se ve en el nomograma.

De este valor sale $m_{h}=1+\frac{4 \times 30 \times 1,54}{200}=1,185$, y entonces:

$$
\frac{P}{\delta}=1,54 \times 30 \times \sqrt{m_{h}}=50 \mathrm{~kg} / \mathrm{m}^{2}
$$

Efectivamente, el peso unitario de las cerchas vale respecto de la sobrecarga:

$$
\frac{p_{h}}{q \cdot S}=\frac{0,8 \times 50}{q}=0,2 \text {, o sea, } m_{h}=1,2 \text {, }
$$

que se aparta de lo tanteado, menos del 1 por 100.

La condición para obtener este costo es que la separación de las cerchas venga dada por la fórmula [2].

Para ello, $\left(\alpha \cdot \frac{R_{h}}{R_{c}}\right)=0,66, N=\frac{L}{V}=20$ correas y, por tanto (del ábaco de la figura 2), $\frac{S}{L}=0,197$, o sea, $S=5,90 \mathrm{~m}$.

Acero A52 para las cerchas: Estudiamos este caso por su interés, y como ejemplo de la utilización de los dos gráficos, cuando se emplean materiales distintos del A37.

En la fórmula [4], para la separación óptima obtenida del ábaco, habíamos prescindido del coeficiente $\mu^{3 / 5}=\left(\lambda \cdot \frac{K_{h}}{K_{c}}\right)^{3 / 5}$.

Para acero A52, el valor de $K_{h} / K_{c}$ es 0,76 . Supuesto el acero de cerchas un 15 por 100 más caro que el de correas (sin incluir taller ni montaje) y según la mano de obra represente el 50 ó el 100 por 100 del costo del acero empleado en las correas, el valor de $\lambda$ oscila entre 
1,075 y 1,10 . Tomando el valor medio $\lambda=1,087$, resulta $\mu^{3 / 5}=0,89$ (lo cual se traduce en que el óptimo buscado consiste en acercar un 10 por 100 más las cerchas que en el caso anterior, con un solo tipo de acero).

En la fórmula [5] para el costo unitario de la estructura se había prescindido de $\mu^{4 / 5}$, que ahora vale 0,86 . Es decir, se consigue un ahorro del 14 por 100, si se emplea este tipo de acero en las condiciones estudiadas.

Por último, la fórmula [8], que daba el peso unitario de la estructura, no da ahora un peso real, por haber afectado del coeficiente $\lambda$ a uno de los sumandos (el 80 por 100 del total). Por tanto, el peso real será, con $\lambda=1,087$, un 6 por 100 menor que el valor de $\frac{P}{\delta}$.

Si no hubiera limitaciones por tolerancia de la flecha y también las correas fueran de A52, entonces $\mu=1, \mathrm{y} \frac{\delta^{\prime}}{\delta}=1,087$, y de [6], $Z^{\prime}=0,72 \cdot Z$, sale $P^{\prime}=0,79 \cdot P$, que representa un ahorro del 21 por 100 en el costo del entramado.

Tubos y secciones rectangulares: Para terminar esta parte dedicada a piezas metálicas daremos una relación de robusteces de tubos, puesto que su uso es creciente para estructuras ligeras, donde sea primordial un montaje rápido, o la posible recuperación del material.

Asimismo, el disponer de un cuadro de robusteces correspondientes a formas geométricas conocidas, resulta muy útil para juzgar de la robustez de una forma poco usual, comparándola con la de estas secciones, que resulta muy intuitiva. Llamando $e$ al espesor y $D$ al diámetro exterior, la relación $D / e$ con respecto a $R$ viene expresada en el cuadro 3:

CUADRO NUM. 3. Robusteces de tubos según su esbeltez relativa

\begin{tabular}{|c|c|c|c|c|}
\hline$\frac{D}{e}$ & 5 & 10 & 20 & 40 \\
\hline$R$ & 1,78 & 1,58 & 1,1 & 0,84 \\
\hline
\end{tabular}

Si han de utilizarse con frecuencia estas secciones y puede haber golpes o cargas no centradas en la estructura, resultará útil construirse otro gráfico, análogo al de la figura 1, para tubos resistentes con el momento admisible y el peso por metro lineal.

La robustez en las vigas de hormigón pretensado: En las secciones metálicas, usuales en las cubiertas de naves, el concepto de robustez sirve como limitación del rendimiento, es decir, actúa como un coeficiente de seguridad a cargas imprevistas. Vamos a ver que no es éste el caso de las vigas de hormigón pretensado, dado que la misma dificultad de ejecución, y puesta en obra de la masa, impiden llegar a las esbelteces propias del acero. Sin embargo, sigue siendo útil el concepto, pues puede fijarse previamente la robustez, igual que en los casos anteriores, atendiendo a que en cada caso resulta una robustez mínima posible $\left({ }^{*}\right)$. La consideración del rendimiento aconseja precisamente utilizar valores mínimos que, por otra parte, coinciden con los usuales en esta técnica del pretensado.

Queremos llamar la atención sobre el trabajo a flexión compuesta del hormigón pretensado. La hipótesis de Navier sigue siendo válida y la capacidad de resistencia $\left(W=\frac{2 \cdot I}{c}=\frac{M}{\sigma}\right)$ en nada se diferencia de las secciones metálicas consideradas hasta ahora.

(*) En vigas de edificación resulta difícil bajar de $R=2$. 
El tensado añade, a la ley de esfuerzos en cada sección, una compresión excéntrica que tendrá como resultado eliminar las tracciones sin aumentar la compresión máxima.

Pero la ley de esfuerzos o deformaciones debida sólo a las cargas (flexión pura) depende de la forma geométrica, exactamente igual que en las secciones homogéneas. Es, por tanto, lineal y está gobernada por la fibra más alejada $\left(\frac{c}{2}\right)$ de la neutra, la carga máxima de trabajo y el reparto de áreas activas (I): La capacidad de trabajo a flexión es idéntica a una viga no tensada, de un material que pudiera resistir tracciones $\left({ }^{*}\right)$.

Para secciones rectangulares $R=3,3 \cdot \sqrt[3]{b / c}$ y las robusteces crecen con el ancho de la viga, pudiendo llegarse a valores muy grandes. Pero obsérvese que valores mayores de 3,3 indican en realidad que la robustez transversal es mayor que la del plano principal y, por tanto, que no tiene ya sentido, a partir de este valor.

En general, la robustez indica capacidad de resistencia a esfuerzos distintos de los principales. Es indudable que su valor debe aumentar indefinidamente al aumentar $b / c$, pero la auténtica debilidad de estas placas estará entonces en su capacidad de soportar momentos principales o cargas de perforación.

Puentes de hormigón pretensado: Una vez fijada una robustez, la fórmula [1] permite obtener una relación entre el peso de la viga y el momento, en función de la separación $S$ entre vigas. En los puentes pretensados, esta separación se fija entre 1,5 y $3,5 \mathrm{~m}$, según el número de calzadas, de modo que prácticamente resulta otro dato. La sobrecarga $q$ para las vigas, consta del peso de la capa de rodadura y del tablero $q_{t}$ (cuando no se juntan las cabezas de las vigas) y del reparto transversal de los trenes de cargas, lo que equivale, en la práctica, a afectar la sobrecarga uniforme equivalente $q_{i}$ de un cierto coeficiente $\vartheta$ tabulable en función de $S$ y de la libertad de tecleo. Cada país establece la $q_{i}$ en función de la luz.

En las normas DIN: $q_{i a}=3,8 / \sqrt{\bar{L}}$ (toneladas/metro cuadrado), y en España $q_{i e}=2,1 \cdot L^{-1 / 5}=$ $=\frac{2,1}{L^{1 / 5}}$ (toneladas $/$ metro cuadrado).

En la figura 4 se han dibujado las escalas que permiten obtener estas sobrecargas, partiendo de la luz. Tenemos, pues:

$$
q=q_{t}+q_{i} \cdot \vartheta .
$$

Análogamente a las secciones metálicas, podemos escribir, para viga apoyada (midiendo $q$ en $\mathrm{kg} / \mathrm{m}^{2}$ con $K_{p}=0,22$ ):

$$
p=K_{p} \cdot R_{p} \cdot M^{2 / 3}=0,22 \cdot R_{p} \cdot(m \cdot q \cdot S)^{2 / 3} \cdot \frac{L^{2 / 3}}{4},
$$

donde:

$$
m=1+\frac{p}{q \cdot S}
$$

$\mathrm{y}$, por tanto, el peso de la viga por $\mathrm{m}^{2}$ de tablero, resulta:

$$
\frac{p}{S}=q \cdot(m-1)
$$

(*) La sección se supone simétrica, pero podría razonarse lo mismo si no lo fuera. 


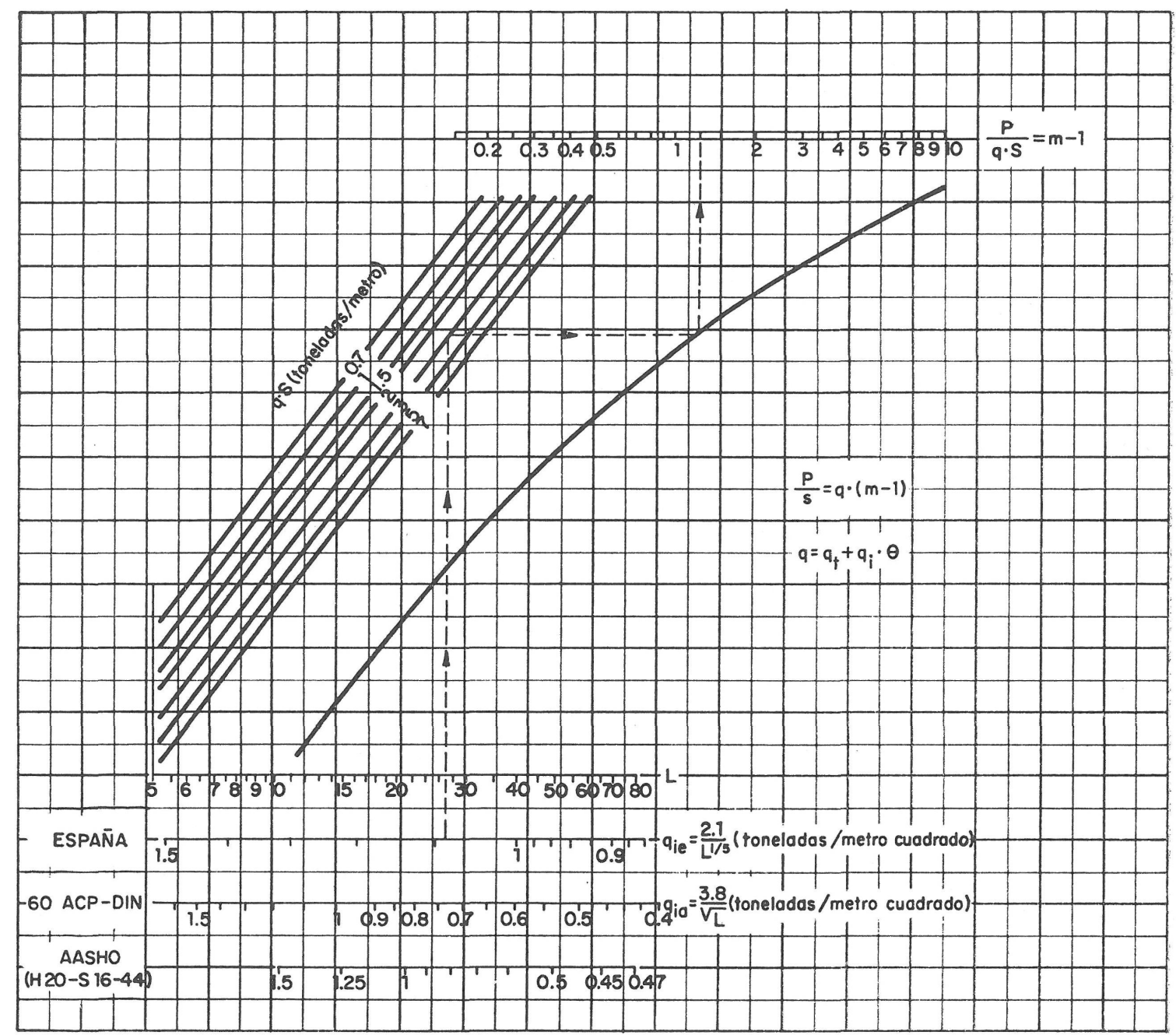

Fig. 4. Abaco para obtentr el costo en los puentes de hormigón pretensado $\left(R_{p}=1,4\right)$.

El valor de $m-1$ sale de [9], escribiendo:

$$
\frac{p}{q \cdot S \cdot m^{2 / 3}}=0,055 \cdot R_{p} \frac{L^{4 / 3}}{(q \cdot S)^{1 / 3}}=\frac{m-1}{m^{2 / 3}}=f(m-1) .
$$

La figura 4 representa esta función para $R_{p}=1,4(*)$. Permite, a partir de la fórmula [10], obtener el peso unitario de cada puente, si se fijan la robustez, la luz y $q \cdot S$ (carga en cada viga).

Como el precio del kilo de hormigón pretensado es casi independiente de los demás datos, en este tipo de grandes vigas, pueden compararse las fórmulas [8] y [10] y estudiar las. soluciones más económicas según la luz y las sobrecargas, sin necesidad de haberlas proyectado.

Este método ha empezado ya a ser utilizado por los autores para informar sobre la tan debatida competencia del hormigón pretensado frente a la solución metálica, según los precios de cada localidad.

(*) Igual que antes, en [6], $f^{\prime}=\frac{f}{1,4} \cdot R_{p}$, cuando se utilice otra robustez que la representada en la fi- 


\section{Le Goefficiemt adimemsionmel de molbustrestese dans le culcul de poutros}

Rafael Escolá, Dr. Ing. industriel.

Oriol Serra, Dr. Ing. des Ponts et Chaussées.

Le concept de «robustesse» est proposé et discuté en tant que mesure de la résistance aux efforts imprévus. Une application est faite à l'espacement le plus économique entre les poutres principales d'un hourdis horizontal pour couvertures ou planchers.

La grande utilité du concept proposé est prouvée par l'obtention directe du prix d'un hourdis résistant, sans avoir à en faire le projet.

Finalement, la robustesse est appliquée au coût de ponts en béton précontraint.

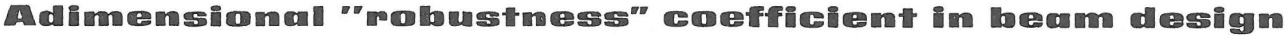

Rafael Escolá, Mechanical Engineer (I. E), Dr. Eng.

Oriol Serra, Civil Engineer (C. E.), Dr. Eng.

The concept «robustness» is defined as a measure of the strength against unforeseen loadings. This idea is applied to the optimal separation of the main beams in a horizontal flooring or roofing framework.

The great usefulness of the proposed coefficient is evident since it is possible to obtain directly the cost of a structural framework, without need to design it in detail.

Finally, the «robustness» coefficient is applied to the cost of prestressed concrete bridges.

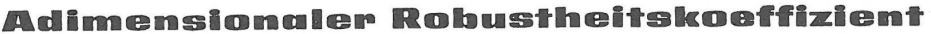 zur Berecthumg vom Barkem}

Dr. Ing. Rafael Escolá.

Dr. Ing. Oriol Serra.

In dieser Arbeit wird der Begriff der Robustheit als Mass der Widerstandsfähigkeit bei unvorhergesehener Beanspruchung erörtert. Er wird für die wirtschaftlichste Entfernung zwischen den Hauptbalken eines Horizontalgerüstes für Dächer und Decken angewendet.

Man verspricht sich hiervon einen grossen Vorteil, da man den Preis eines widerstandsfähigen Geruistes sofort, ohne Voranschlag, errechnen kann.

Schliesslich wird die Robustheit für die Kosten von Bricken aus Spannbeton angewendet. 